\title{
Design Of Its Through IoT
}

\author{
R. Velmani, A. Srinivasa reddy, M. Ranjith Reddy
}

\begin{abstract}
Due to the population and economic growth, the vehicle population is increased in India and that creates a critical burden on traffic management in metropolitan cities (Hyderabad, Bangalore, Chennai, Mumbai, etc.) and towns of the country. This paper proposes Intelligent Transportation System (ITS) by using information and communication technologies like IoT and data analytics and that apply to transport operations. ITS aimed to create safe, efficient, reliable and environmentally sustainable smart transport systems. At every traffic signal the density of the traffic is detected with the help of IR sensors, then the traffic level is obtained. The IR sensors are connected to 8051 microcontroller. A mobile data service is connected to microcontroller to deliver the real time traffic information through IoT to the traveller, so that he can know the traffic level and can take an alternate. The information gathered from the traffic signals is processed with the help of Big Data Analytics and the data is to be sent to every traveller's to make their journey safe and reach their destination in time.
\end{abstract}

Keywords :- Intelligent transport systems, Internet of things, ITS node, traffic signals, end-to-end communication.

\section{INTRODUCTION}

ITS plays an major role to develop smart cities. Since, the public transportation system is the essential source of travel for many people's living in the urban as well as rural for many developing countries. ITS was first stated in US and spreaded to other countries, which enables real-time, efficient and accurate traffic management system to offer the service with maximum efficiency. Due to the application growth of internet, sensor technology and communication network devices, the ITS based IoT has a great potential development in couple of years [1-4].

ITS is a large-scaled informative, intelligent, socially integrated management system, which uses latest technologies such as sensor, real-time information, wireless network, image processing, computing, automatic control and so on to the whole system. The main advantages of ITS through IoT are low cost, reliable, easy to access, safe journey and reach the destination in time.

The applications of the ITS include intelligent public transport system, mobile emergency command and scheduling management system, commercial vehicle functional system, non-stop electronic highway toll paying system, travel and traffic management system, travel demand administration system, advanced vehicle control and safety management systems, e-portal, vehicle anti-theft

Revised Manuscript Received on April 19, 2019.

R. Velmani, Department of Electronics and Communication Engineering, Siddhartha Institute of Technology \& Sciences, Hyderabad, Telangana, India.(Email: velmanir@gmail.com)

A. Srinivasa reddy, Department of Electronics and Communication Engineering, Siddhartha Institute of Technology \& Sciences, Hyderabad, Telangana, India.

M. Ranjith Reddy, Department of Electronics and Communication Engineering, Siddhartha Institute of Technology \& Sciences, Hyderabad, Telangana, India. system, transportation or highway police mobile law enforcement, etc.

\section{RELATED WORK}

The IoT plays an major role in ITS, since, IoT exchanges the real time information between conveyances and highway roads, and in between to conveyances to conveyances. The IoT is an emerging network, which is associated with various types of devices to share and exchange the information from remote location such as Radio Frequency IDentification (RFID), laser scanner, infrared (IR) sensors, Global Position System (GPS), etc., through an inter network connection [5-7].

Usually, embedded devices are designed to perform a few pre-defined tasks and that is fully automated and dedicated to specific requirements. Even embedded devices are designed for limited operations, the design engineers can improve the performance of the device as well as to reduce the size and price of the product. Embedded systems are manufactured in large scale and that benefited to economic scale. Due to the nature of hardware design, Personal Digital Assistants (PDAs) or handheld devices are usually considered to be an embedded devices and that can be expandable in software terms. Embedded device design research is familiar with potential contents since it combine the software and hardware design challenges. In IoT based ITS, the embedded devices are playing an major role on end-to-end real-time communication.

\section{INTELLIGENT TRANSPORTATION SYSTEM THROUGH INTERNET OF THINGS}

Fig. 1 shows the proposed architecture of ITS based IoT. Various embedded devices are considered to perform simultaneous operations in that proposed architecture.

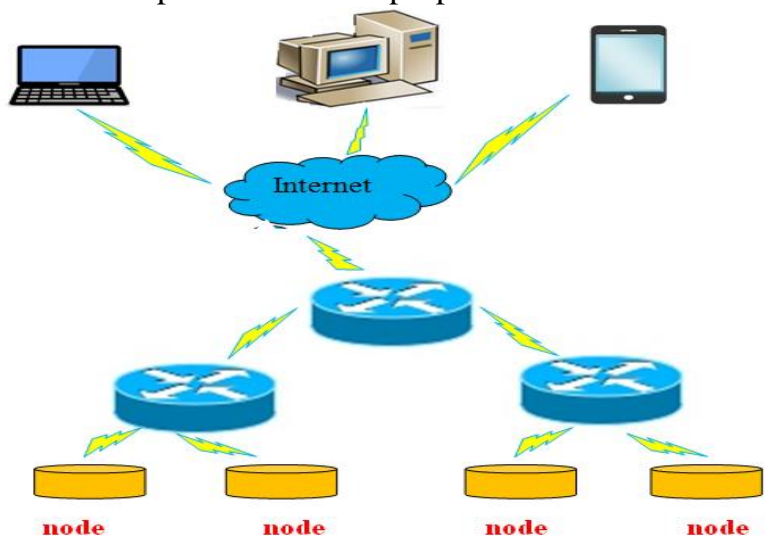

Fig. 1 The Structure of Intelligent Transport System

Published By:

Blue Eyes Intelligence Engineering

\& Sciences Publication 
The major components of the ITS based IoT architecture are ITS nodes, gateway and mobile data services. Here, the nodes are considered as an embedded devices which collects the physical quantity of information and that will be converted as a digital data. Then, the mobile data service helped to forward that real-time data to the remote devices.

\subsection{ITS nodes}

ITS nodes form the brain network structure for that intelligent transportation management system. Hardware structure of the ITS node is shown in the Fig. 2. The proposed ITS nodes help to measure various physical phenomena such as light, temperature, vehicle speed, humidity, pressure, vehicles distance, etc. Sensors in a ITS node sense the real-time phenomena. Multiple sensors are used on that particular ITS node to collect a very specific real time information, and that sensed data will be forwarded immediately to the remote user through an internet connectivity.

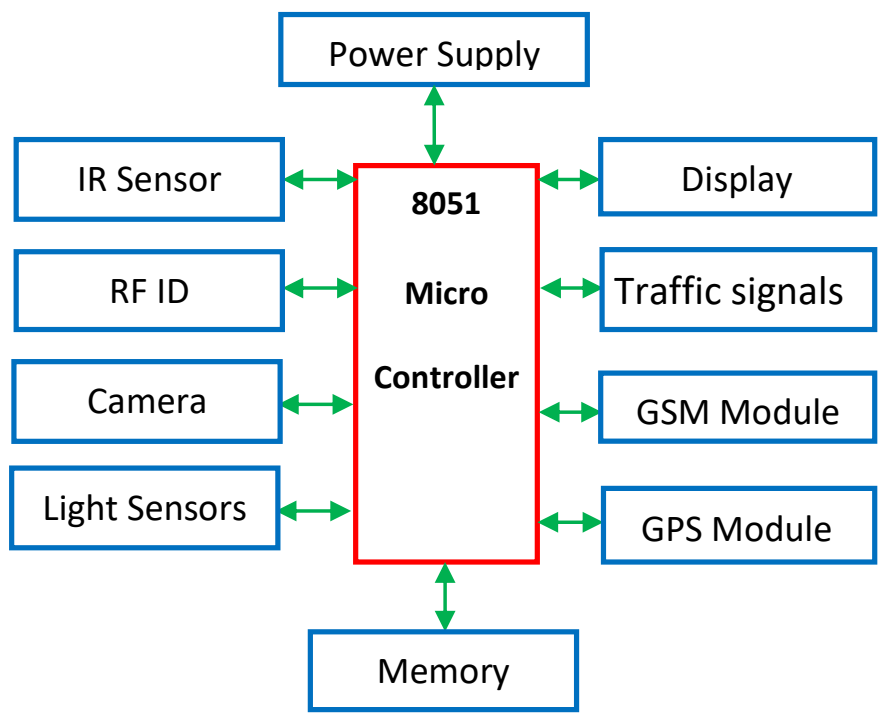

Fig. 2 ITS Node Hardware Structure.

Here, the ITS nodes are helped to control the speed of vehicles on traffic signals. Further, the gateway plays an important role to collect the information from the ITS nodes and that forward the real time data to the remote user through an internet. Here, the internet services help to extract the information about the vehicles mobility, track changes, traffic conditions, automatic toll payment, etc., to the remote destination. The major components of the ITS nodes are 8051 microcontrollers, location identifier, mobile data service, RFID, IR sensors, memory, power supply, camera, traffic signals and display.

\section{(a) 8051 microcontrollers}

Generally, microprocessors and microcontrollers are electronic components. Mostly, they are used in real time and embedded device based application products. Microcontroller is a real time programming device. It consists of a Central Processing Unit (CPU) with a number of Random Access Memory (RAM), Input/Output ports, Read Only Memory (ROM) and a timer, which are embedded as a single chip. Here, the number of components may define the cost and space of that device. Intel corporation developed a single chip microcontroller $(\mu \mathrm{C})$ in 1980 's and popular in early 1990's, i.e., Intel $8051 \mu \mathrm{C}$.

Nowadays, 20 independent of 8051 enhanced devices are manufactured by including Atmel corporation, Infineon Technologies AG, Maxim Integrated Products, etc. 8051 microcontroller having a 8-bit processor, and that can process of 8 bits real time data at a moment in time. More than 8 bits real time data can be broken into 8-bit pieces and that will be deal with CPU. Also, it is having different types of memory such as UltraViolet-Erasable Programmable Read-Only Memory (UV-EPROM), NonVolatile-Random Access Memory (NV-RAM) and Flash. The AT89S52 is a 8051 microcontroller based full static Complementary Metal Oxide Semiconductor (CMOS) controller with a 3 level program memory lock, $8 \mathrm{~K}$ flash memory, 32 input/output lines, 8 interrupts sources, 3 timers/counters, watchdog timers, 2 Data Pointer Registers (DPTRs) and 256 byes Onchip RAM. On-chip flash memory will allow to program or reprogram the coding of the system.

\section{(b) Location identifier}

Location identifier identifies the current location of the vehicles. Global Position System (GPS) receiver is used to capture the National Marine Electronics Association (NMEA) sentence from the 24 GPS satellites positioned in 6 earth-central orbital planes with 4 operated satellites, which can find few parameters such as velocity, latitude, longitude, altitude, time stamp, etc.

\section{(c) Mobile data service}

In ITS, mobile data service or GSM module (i.e., $3 \mathrm{G} / 4 \mathrm{G} / \mathrm{LTE}$ ) is added to forward the real-time data to the remote user via the internet, which utilizes mobile frequency band such as 850/900/1800/2100/2300/2500 MHZ.

\section{(d) RFID}

RFID is an electronic device. It uses the radio wave to automatically interpret and capture the previously stored information on a tag which is coupled to the object. The tag can be read by the reader without any Line of Sight (LoS) signal up to several feet away. In RFID device, tag and label are fixed together with the transmitter and receiver. Also, the tag has a microchip and an antenna, and a specific serial number for an object. Two types of RF ID tags are available. i.e., active and passive. Active tags are incorporated with a tiny battery which enables the relay information exchange. Passive tags use an interrogator's electromagnetic energy to enable the relay by the stored information.

\section{(e) IR Sensors}

An IR sensor is an electronic component which emit or detect the characteristics (i.e., infrared radiation) of the surroundings. Also, infrared sensors are capable to measure the heat energy which is emitted by an object or detect the motion of the object. Since, all objects may radiate the thermal energy and that is not visible to human eyes. But, the thermal energy can be detected by an infrared sensor. The wavelength of infrared waves between 0.75 and 
$1000 \mu \mathrm{m}$. Usually, the emitter is an IR LED and the detector is an IR photodiode. The photodiode is very sensitive to IR light with respect to the IR LED light. When the IR light falls on the photodiode, the resistance and the voltage output is varied in propagation to the magnitude of the received IR light. Active and passive are the two types of infrared sensors. Active infrared sensors emit the infrared radiation by the IR LED and the photodiode or photoelectric cells or phototransistor will receive the reflected light. Active infrared sensors simply detect the infrared radiations from the external source (i.e., the sensor provide a reading based on the thermal input).

\section{(f) Display}

Liquid Crystal Display (LCD) module is an electronic display screen which consists of 2 lines. Here, each line may have 16 characters and that each character consists of $5 \times 8$ pixel matrixes. LCD has 2 registers namely data and command. The data register fetches the temporary data and that will be displayed on LCD screen. Also, the command register stores the command instructions given to the LCD. Light Emitting Diode (LED) is a electronic semiconducting device which emits a visible light energy when an electric potential passing across it. LED consists of $p$-type and ntype processed semiconductor materials, and that has a limpid package which allows the light or infrared energy to passing on it.

\section{CONCLUSION AND FUTURE SCOPE}

Since, the vehicle traffic management is a major issue in metropolitan cities, ITS enabled on IoT concept is very useful for the development of the nation's pride, by reducing the traffic rate in peak hours, in order to reach the destination safe and within time. This method is very useful on easy transportation and emergency situations like ambulance and fire engine to reach the destination in time to save the life, since, it controls the traffic pattern in every junction up to an extent in future as widespread. Since, the proposed work is very useful to monitor the vehicle traffic in urban areas, each traveller can get the traffic information towards to destination via internet without any destruction or disturbance.

\section{REFERENCES}

1 A.S. Salim and M.I. Ibrahim, "Design and Implementation of Web-Based GPS-GPRS Vehicle Tracking system", International Journal of Computer Science and Information Technologies, vol. 3, no. 12, pp. 443-448, 2013.

2 V. Karagiannis and P. Chatzimisios and F. VazquezGallego and J. Alonso-Zarate "A Survey on Application Layer Protocols for the Internet of Things", Transaction on IoT and Cloud computing, vol. 1, no. 1, 2015.

3 V. Lelitha, R. Gitakrishnan and A. Anand, "Intelligent Transportation Systems - Synthesis Report and Issues and Challenges Under Indian conditions", Centre of Excellence in Urban Transport, IIT Madras, pp. 1-57, December 2010.

4 T.M. Bojan, U.R. Kumar and V.M. Bojan, "An internet of things based intelligent transportation system", IEEE International Conference on Vehicular Electronics and Safety, Hyderabad, 2014, pp. 174-179. DOI: 10.1109/ICVES.2014.7063743.
5 H.Y. Feng, J.L. He, L.J. Zhang and F.G. Meng "An Application of RFID Technology in Traffic Congestion Management", Applied Mechanics and Materials, vol. 513-517, pp. 3954-3957, 2014.

6 H. Lingling, L. Haifeng, X. Xu and L. Jian,, "An Intelligent Vehicle Monitoring System based on Internet of Things", 2011 Seventh International Conference on Computational Intelligence and Security, Hainan, 2011, pp. 231-233. DOI: 10.1109/CIS.2011.59.

7 Z. Xu, J. He and Z. Chen, "Design and actualization of IoT-based intelligent logistics system," 2012 IEEE International Conference on Industrial Engineering and Engineering Management, Hong Kong, 2012, pp. 22452248. DOI: 10.1109/IEEM.2012.6838146 